\title{
Travel Grants to Learned Societies' Meetings
}

CPSA-Annual General Meeting, University of Calgary, Calgary, Alberta, June 12-14, 1994

The Canadian Political Science Association receives a travel grant from The Social Sciences and Humanities Research Council of Canada to assist MEMBERS to come to the annual meeting from distant places in Canada. These funds are particularly designed to help participating junior members. They are all apportioned by May 1 st before the meetings take place.

The assumption remains that established faculty who are participating as paper-givers, discussants or chairpersons will be subsidized by their own university. Planners of the programme must inform participants of the deadline.

To be considered, requests for travel funds must reach The Secretary-Treasurer, Canadian Political Science Association, \#205-1 Stewart Street, Ottawa, Ontario K1N 6H7 by March 15, 1994.

Your letter of application must state: (1) that you have approached your own institution for funds; (2) the amount of any partial grants you expect to receive from other sources than the CPSA; (3) your academic rank; (4) your programme function.

\section{Indemnité de déplacement pour le congrès des sociétés savantes}

ACSP-Réunion annuelle générale, Université de Calgary, Calgary, Alberta, 12-14 juin 1994

L'Association canadienne de science politique reçoit des fonds du Conseil de recherches en sciences humaines du Canada pour défrayer les frais de déplacement des MEMBRES résidant dans les régions du pays qui sont loin de la conférence. Ces subventions s'adressent avant tout aux membres-participants les moins établis. Les bénéficiaires seront tous désignés (au ler mai) avant la réunion.

On a tout lieu de croire que les professeurs qui participeront, soit comme conférenciers, rapporteurs ou présidents, recevront l'aide de leur université. Nous soulignons la date limite suivante aux organisateurs du programme qui doivent en aviser les participants.

Prière d'adresser toute demande de subvention au Secrétairetrésorier, Association canadienne de science politique, \#205-1 rue Stewart, Ottawa, Ontario K1N 6N7, avant le 15 mars 1994.

Votre lettre de demande doit mentionner: (1) que vous avez solicité une subvention de votre université; (2) le montant de toute subvention que vous pensez recevoir par ailleurs; (3) votre rang universitaire;

(4) votre rôle au congrès (présentation d'une communication...). 